\title{
3D-printed Electrochemical Sensors: A new horizon for measurement of biomolecules
}

\author{
Aya Abdalla ${ }^{1,2}$ and Bhavik Anil Patel ${ }^{1,2 *}$ \\ ${ }^{1}$ School of Pharmacy and Biomolecular Sciences and ${ }^{2}$ Centre for Stress and Age- \\ Related Diseases, University of Brighton, Brighton, BN2 4GJ, UK
}

${ }^{*}$ Corresponding authors

E-mail address: B.A.Patel@brighton.ac.uk

\begin{abstract}
Electrochemical sensors are widely used to monitor biomolecules. However, limitations in sensor geometry has restricted the scope of currently utilised electrochemical sensors. 3D-printing has emerged as a promising manufacturing approach, to robustly make electrochemical sensors, that can stably measure in biological environments. This review highlights the recent trends in the development of 3D-printed electrodes and biosensors for measurement of biomolecules. Novel geometries of 3D-printed electrodes have provided the means to conduct ex vivo measurement in the intestinal tract and in vivo measurements in the brain. 3D-printing is providing the ability to manufacture electrochemical sensors that can measure biomolecules in diverse areas of the body.
\end{abstract}

\section{Keywords}

Additive manufacturing, 3D-printing, electrochemistry, bioanalysis, neurotransmitters 


\section{Introduction}

Measurement of biomolecules is of significant interest, given the important roles they play in governing physiological functions. Changes in the levels of biomolecules often occur during the onset of disease. Hence, any approach capable of measuring the concentration of biomolecules can be utilised as vital diagnostic technology. However, measurement of biomolecules is a significant challenge, as these molecules are released rapidly in small concentrations from specific locations within a complex environment [1]. Electrochemical techniques have emerged as the leading approach to meet such complex measurement needs [2]. Due to the diverse nature of environments in which measurements are conducted, a wide range of electrochemical sensors have been developed for biomolecular detection [3, 4]. However, the fixed geometry of these electrodes, reduce their scope for bioanalytical monitoring. At present the range of geometries that electrodes are made of are limited and often electrochemical sensing devices are not fabricated to interface with the biological environment. There is also a limitation in the range of conductive materials that can be used to fabricate electrodes. Therefore, complex modifications of the electrode surface are conducted to enhance its electrochemical activity for detection of specific biomolecules. To overcome these limitations and enhance the horizon in which electrochemical measurement can be used for biomolecular detection, 3D-printing has risen as the potential alternative. Its use in the generation of electrochemical sensors and devices can broaden the types of biomolecules detected in varying biological environments. This review will explore the range of sensors that have been fabricated using 3D-printing for biomolecular measurement and the potential opportunities this offers for future measurements in biological environments.

3D-printing is an approach in which digitally generated design can be manufactured into a physical object by printing [5]. 3D-printing of electrodes is most widely conducted using fused deposition modelling (FDM) through the use of commercially available conductive thermoplastic [6-8]. In this approach, conductive thermoplastic is heated to 
a semi-molten state and extruded at a dispenser nozzle. The extruded conductive thermoplastic is deposited layer by layer to generate the 3D-structure. The resulting sensor is either used as manufactured, or pre-treated through different mechanical, chemical, electrochemical, and biological methods to enhance its selectivity to specific molecules [9-12]. These different pre-treatment methods are used to enhance the conductivity and electrochemical performance of 3D-printed sensors to make them suitable for biological measurements.

\section{The potential of 3D-printed electrodes as biosensors}

Electrochemical biosensors are chemical sensors where a biological macromolecule acts as a sensing element. This element is coupled to an electrode, for the purpose of detecting the concentration or activity of a specific analyte in a sample matrix. A variety of biological macromolecules have been utilised within biosensors such as nucleic acids, antibodies and enzymes, of which the latter is the most widely used biological sensing element [13].

The La Belle group printed graphene/PLA sensors onto a mylar substrate which was then dipped into a glucose dehydrogenase solution to make the first ever electrochemical 3D-printed glucose biosensor [14]. Another type of enzymatic biosensor immobilized horseradish peroxidase onto the surface of graphene/PLA sensors. This sensor was able to selectively detect peroxide in human serum and was stable for a period of 7 days [15].

Another important advance is the development of a DNA biosensor. A 3D-printed helical electrode was electrodeposited with gold and thiol single stranded DNA which was attached to the surface of the electrode. The biosensor showed exceptional promise to identify complementary DNA in the presence of non-complementary DNA (Figure 1) [16].

The ability for biological entities to be stable on 3D printed electrodes indicates that they are biocompatible. Therefore 3D-printing provides an economical and robust alternative to current strategies for biosensor fabrication. However, to fully understand the potential 
of these types of biosensors, a comparison to other approaches for biosensor manufacture is critical.

\section{Interfacing 3D-printed electrodes in biological fluids}

Biological fluids are complex measuring matrices due to the presence of a variety of large proteins, which foul the electrode surface [17]. This poses a signficant challenge for conducting stable electrochemical measurements over time.

The Bonacin group utilised a pre-treated graphene/PLA electrode for the measurement of key neurotransmitter dopamine. They were able to monitor dopamine in human blood serum spiked samples with good recovery [11]. Their sensors were reproducible, selective against uric acid and ascorbic acid, which are common biological interferents, and had low limits of detection which is critical in measurements in biological environments. However, the measurement of this molecule without time consuming activation of the surface is difficult. One group solved this by manufacturing their own graphene loaded PLA filament and showcased that untreated 3D printed electrode has superior performance to glassy carbon electrodes for the measurement of dopamine [18].

Using graphene/PLA electrode in a fully 3D printed electrochemical cell, simultaneous detection of nitrite and uric acid in pure and spiked samples of human urine and saliva was achieved [19]. The same electrochemical flow cell was utilised, where the graphene/PLA electrode was modified to an enzymatic biosensor for the detection of glucose, where measurements were conducted in spiked samples of bovine plasma. This 3D printed biosensor was highly stable and showed no interference from sodium, potassium or urea, which are commonly found in blood plasma [20].

These studies show that 3D electrodes can conduct robust measurements in complex biological fluids such as plasma, urine and saliva. These studies indicate that 3D-printed sensors can be an ideal platform for monitoring biomolecules in harsh biological fluid samples. 


\section{Ex vivo and In vivo measurements using 3D-printed sensors}

Measurements of biomolecules ex vivo and in vivo pose a significant challenge due to the restricted geometries required to interface with tissues, large number of interferences, the small concentrations of biomolecules released and the dynamic nature of measurements [21, 22]. Two studies that overcame these challenges were accomplished by the Patel group where in one a 3D-mould packed with carbon composite electrodes was developed. This was utilised for detection of serotonin overflow from the entire murine colon [23]. This approach highlighted the ability to use 3D-printing to generate devices that provided the means to monitor biomolecules from large tissue regions. The second study showed the first ex vivo tissue study conducted with a completely FDM 3D-printed electrochemical sensor. The electrode was uniquely manufactured to mimic the shape of a feacal pellet, which is critical in order to provide a natural physiological stimulus. This 3D-printed device was then inserted into the lumen of the anorectum in order to simultaneously detect serotonin overflow and circular muscle contractility (Figure 2) [24]. This study directly shows that 3D-printing provides a beneficial platform to create varying electrode geometries that are best suited to naturally interface with biological systems.

Although most studies reviewed so far have been conducted with FDM, this mode of 3D-printing is not ideal for development of sensors with sub-micron dimensions. Using two-photon lithography methods, various studies have developed microelectrodes. A study by the Venton group used this approach, where two-photon lithography was used to 3D-print photoresist on the tip of metal electrodes. This was then carbonized to create sphere microelectrodes [25]. Figure 3 shows these 3D-printed electrodes being utilised for in vivo detection of dopamine in vivo in the caudate [26]. Dopamine release as low as $92 \mathrm{nM}$ was successfully detected in vivo. This approach provides the ability to 
revolutionize electrode fabrication by creating electrodes of varying geometries for implantable sensors.

\section{Conclusions \& outlook}

3D-printed electrodes have shown the ability to conduct sensitive and robust measurements of biomolecules and as biosensors. Given that 3D-printing provides the scope to generate sensors of varying shapes and sizes, novel electrode geometries have been fabricated so that they can interface with biological environments.

The outlook for biomolecular detection is highly promising. 3D-printed electrochemical sensors can be made with any dimension and geometry, allowing for sensors to be interfaced in any biological system. Additionally, 3D-printable filaments can be made using a wide array of conductive materials, opening new avenues for biomolecular detection. Furthermore, the ability to have such complex devices made at scale in a robust fashion allows added benefits. With all these potential benefits, the scope of 3Dprinted electrodes for providing new insight into the role and function of biomolecules is profound. Future medical diagnostic devices could be completely additively manufactured, where electrochemical sensors could serve as the detection technology.

\section{Conflict of interest statement}

None

\section{Acknowledgements}

The authors like to thank CRUK ESPRC Multidisciplinary Project Award (C57783/A24316; NS/A000065/1) for funding. We would like to thank Emily Brooks, Mareike Herrmann and Ana Tendero Canadas for reviewing the contribution. 


\section{References}

Papers of particular interest, published within the period of review have been highlighted as:

${ }^{*}$ special interest

**outstanding interest

[1] R.M. Wightman, Probing Cellular Chemistry in Biological Systems with Microelectrodes, Science 311(5767) (2006) 1570-1574.

[2] K.L. Adams, M. Puchades, A.G. Ewing, In Vitro Electrochemistry of Biological Systems, Annual Review of Analytical Chemistry 1(1) (2008) 329-355.

[3] M.L. Huffman, B.J. Venton, Carbon-fiber microelectrodes for in vivo applications, Analyst 134(1) (2009) 18-24.

[4] O.D. Renedo, M.A. Alonso-Lomillo, M.J.A. Martínez, Recent developments in the field of screen-printed electrodes and their related applications, Talanta 73(2) (2007) 202-219.

[5] W.K. Swanson, S.D. Kremer, Three dimensional systems, Google Patents, 1978.

[6] A. Ambrosi, M. Pumera, 3D-printing technologies for electrochemical applications, Chem Soc Rev 45(10) (2016) 2740-2755.

[7] C.L. Manzanares Palenzuela, F. Novotný, P. Krupička, Z. Sofer, M. Pumera, 3DPrinted Graphene/Polylactic Acid Electrodes Promise High Sensitivity in Electroanalysis, Analytical Chemistry 90(9) (2018) 5753-5757.

[8] M. Pumera, Three-dimensionally printed electrochemical systems for biomedical analytical applications, Current Opinion in Electrochemistry 14 (2019) 133-137. 
[9] R.M. Cardoso, C. Kalinke, R.G. Rocha, P.L. dos Santos, D.P. Rocha, P.R. Oliveira, B.C. Janegitz, J.A. Bonacin, E.M. Richter, R.A.A. Munoz, Additive-manufactured (3Dprinted) electrochemical sensors: A critical review, Anal Chim Acta (2020).

[10] R. Gusmão, M.P. Browne, Z. Sofer, M. Pumera, The capacitance and electron transfer of 3D-printed graphene electrodes are dramatically influenced by the type of solvent used for pre-treatment, Electrochemistry Communications 102 (2019) 83-88.

*[11] C. Kalinke, N.V. Neumsteir, G.d.O. Aparecido, T.V.d.B. Ferraz, P.L. dos Santos, B.C. Janegitz, J.A. Bonacin, Comparison of activation processes for 3D printed PLAgraphene electrodes: electrochemical properties and application for sensing of dopamine, Analyst 145(4) (2020) 1207-1218.

[12] P.L. dos Santos, V. Katic, H.C. Loureiro, M.F. dos Santos, D.P. dos Santos, A.L.B. Formiga, J.A. Bonacin, Enhanced performance of 3D printed graphene electrodes after electrochemical pre-treatment: Role of exposed graphene sheets, Sensors and Actuators B: Chemical 281 (2019) 837-848.

[13] P. Mehrotra, Biosensors and their applications - A review, Journal of Oral Biology and Craniofacial Research 6(2) (2016) 153-159.

**14] A. Adams, A. Malkoc, J.T. La Belle, The Development of a Glucose Dehydrogenase 3D-Printed Glucose Sensor: A Proof-of-Concept Study, Journal of diabetes science and technology 12(1) (2018) 176-182.

**First ever electrochemical 3D-printed glucose biosensor that functions at physiological levels

*[15] A.M. López Marzo, C.C. Mayorga-Martinez, M. Pumera, 3D-printed graphene direct electron transfer enzyme biosensors, Biosensors and Bioelectronics 151 (2020) 111980.

**[16] A.H. Loo, C.K. Chua, M. Pumera, DNA biosensing with 3D printing technology, Analyst 142(2) (2017) 279-283.

\section{**First 3D printed DNA biosensors, showcasing potential for interfacing biological elements with 3D printed surfaces}

[17] N. Wisniewski, M. Reichert, Methods for reducing biosensor membrane biofouling, Colloids and Surfaces B: Biointerfaces 18(3-4) (2000) 197-219. 
[18] R.M. Cardoso, D.M.H. Mendonça, W.P. Silva, M.N.T. Silva, E. Nossol, R.A.B. da Silva, E.M. Richter, R.A.A. Muñoz, 3D printing for electroanalysis: From multiuse electrochemical cells to sensors, Analytica Chimica Acta 1033 (2018) 49-57. ** [19] R.M. Cardoso, P.R.L. Silva, A.P. Lima, D.P. Rocha, T.C. Oliveira, T.M. do Prado, E.L. Fava, O. Fatibello-Filho, E.M. Richter, R.A.A. Muñoz, 3D-Printed graphene/polylactic acid electrode for bioanalysis: Biosensing of glucose and simultaneous determination of uric acid and nitrite in biological fluids, Sensors and Actuators B: Chemical 307 (2020) 127621.

\section{** Glucose, nitrite, and uric acid measurements made in human urine and saliva}

[20] V. Katseli, A. Economou, C. Kokkinos, A novel all-3D-printed cell-on-a-chip device as a useful electroanalytical tool: Application to the simultaneous voltammetric determination of caffeine and paracetamol, Talanta 208 (2020) 120388.

[21] K. Štulík, Challenges and Promises of Electrochemical Detection and Sensing, Electroanalysis 11(14) (1999) 1001-1004.

[22] T. Xiao, F. Wu, J. Hao, M. Zhang, P. Yu, L. Mao, In Vivo Analysis with Electrochemical Sensors and Biosensors, Analytical Chemistry 89(1) (2017) 300-313.

[23] N. Patel, A. Fagan-Murphy, D. Covill, B.A. Patel, 3D Printed Molds Encompassing Carbon Composite Electrodes To Conduct Multisite Monitoring in the Entire Colon, Analytical Chemistry 89(21) (2017) 11690-11696.

${ }^{*}$ [24] H.H. Hamzah, O. Keattch, M.S. Yeoman, D. Covill, B.A. Patel, ThreeDimensional-Printed Electrochemical Sensor for Simultaneous Dual Monitoring of Serotonin Overflow and Circular Muscle Contraction, Analytical Chemistry 91(18) (2019) 12014-12020.

\section{${ }^{* *}$ First ex vivo measurement using a completely 3D printed electrochemical sensor}

[25] E. Trikantzopoulos, C. Yang, M. Ganesana, Y. Wang, B.J. Venton, Novel carbonfiber microelectrode batch fabrication using a 3D-printed mold and polyimide resin, Analyst 141(18) (2016) 5256-5260.

** [26] C. Yang, Q. Cao, P. Puthongkham, S.T. Lee, M. Ganesana, N.V. Lavrik, B.J. Venton, 3D-Printed Carbon Electrodes for Neurotransmitter Detection, Angewandte Chemie International Edition 57(43) (2018) 14255-14259. 
${ }^{\star \star}$ First in vivo measurement using 3D manufactured microelectrodes using twophoton polymerization 
FIGURE 1. Development of a biosensor on a 3D-printed electrode (A) Schematic representation of the DNA biosensor developed on gold electroplated 3D-printed helical stainless-steel electrodes. (B) Graph showing the methylene blue reduction peak current values obtained after hybridization with complementary or non-complementary DNA targets. The inset demonstrates the respective differential pulse voltammograms acquired. Reprinted with permission from Ref. [16]
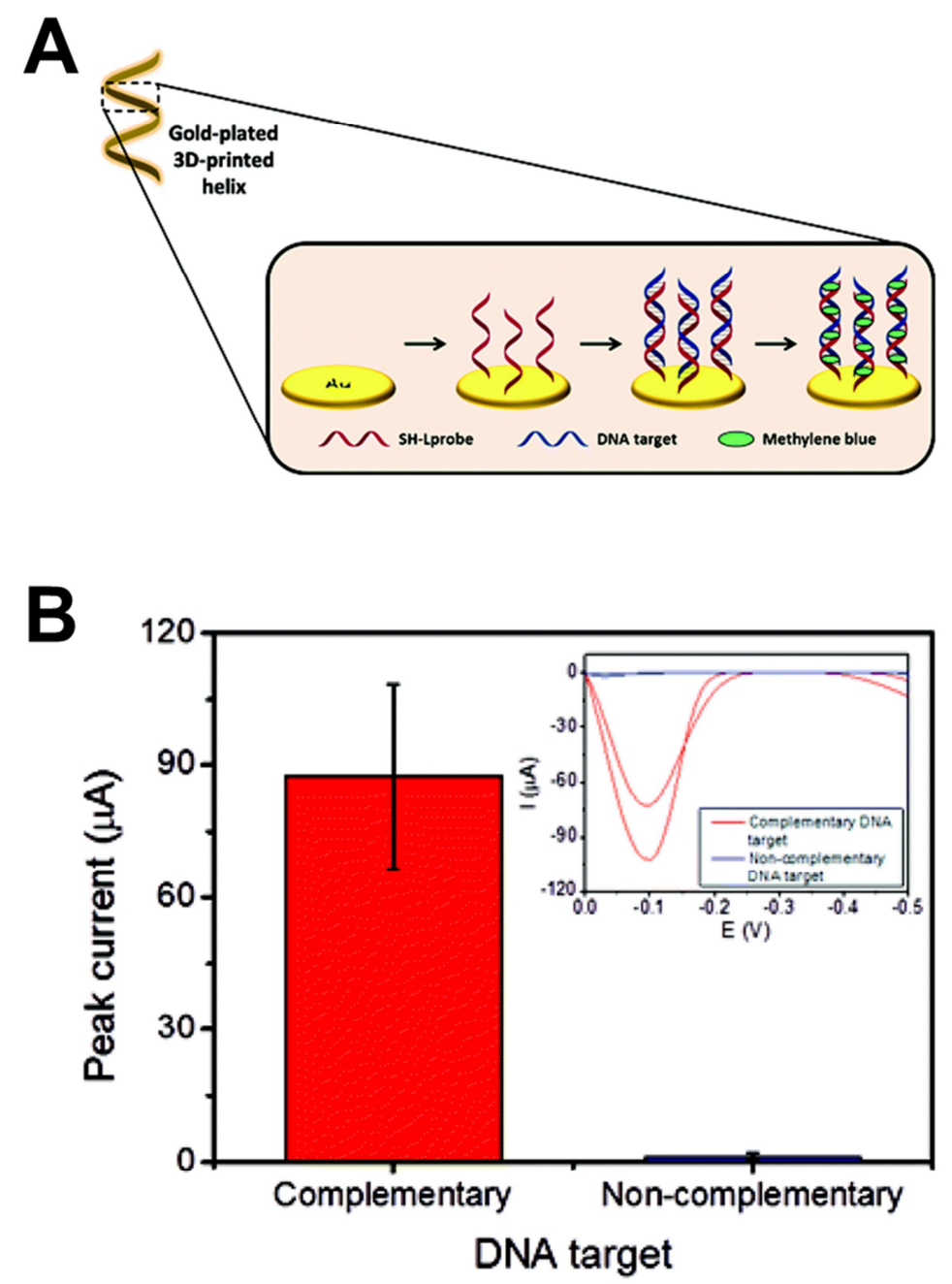
FIGURE 2 Fabrication of 3D-printed electrochemical sensor. (A) Schematic visualization of the electrochemical sensor, showcasing how connection of the sensor was achieved. (B) Final 3D-printed electrochemical sensor with a geometry that mimics a faecal pellet, where (1) is the carbon black/PLA electrode and (2) is the PLA sealing cap. (C) Simultaneous monitoring of 5-HT overflow and circular contraction from the anorectum, where recordings on the force transducer (red) and electrochemical sensor (blue) are shown. Gray bar indicates the duration that $1 \mu \mathrm{M}$ fluoxetine was added. Reprinted with permission from Ref. [24]
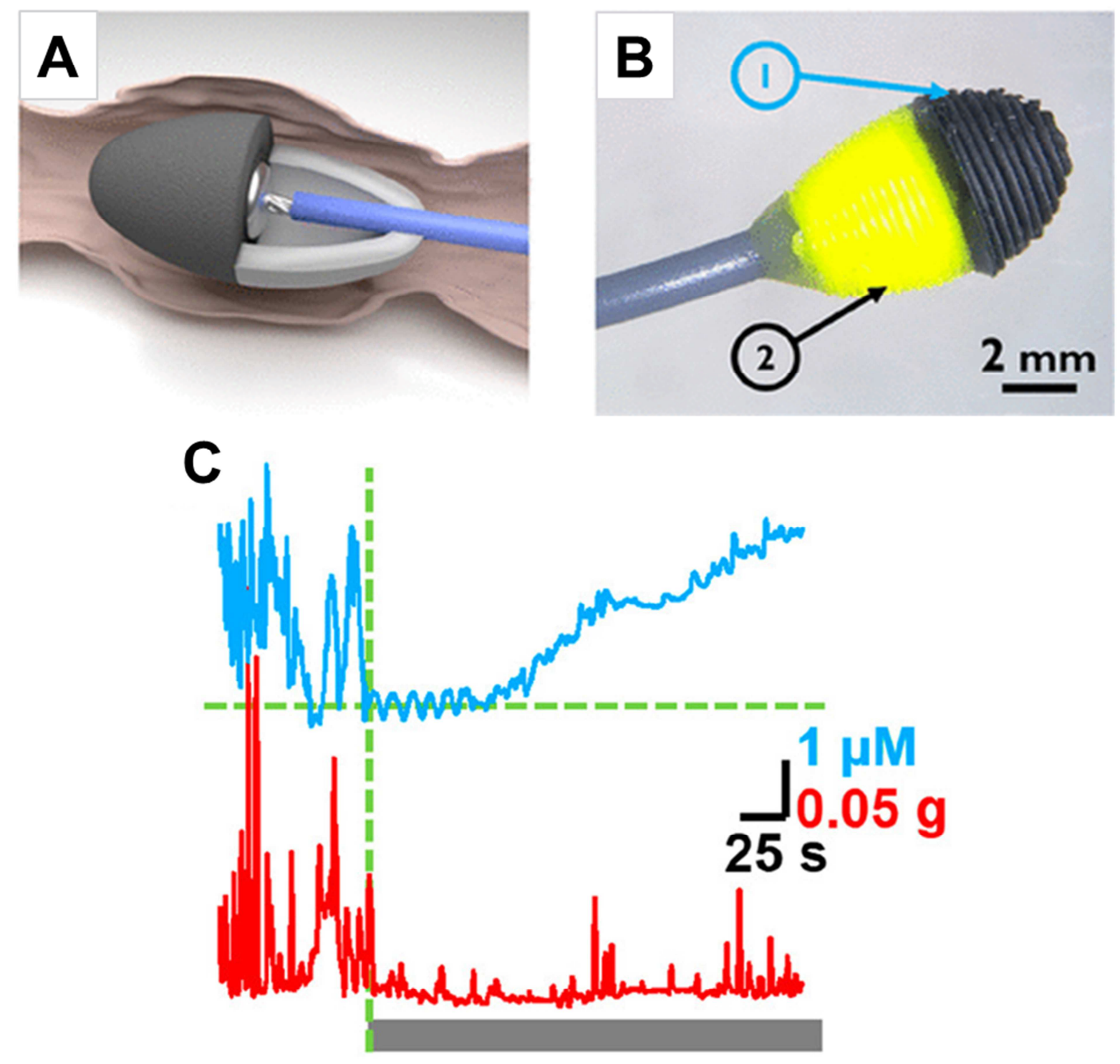
FIGURE 3. 3D-printed microelectrodes for detection of dopamine in vivo. SEM images of 3D-printed sphere (A) before and (B) after pyrolysis. (C) Evoked dopamine current versus time (60 stimulation pulses, $60 \mathrm{~Hz}, 300 \mu \mathrm{A}$ biphasic stimulation pulses applied at $5 \mathrm{~s})$. (D) Cyclic voltammogram of stimulated dopamine. Reprinted with permission from Ref. [26]
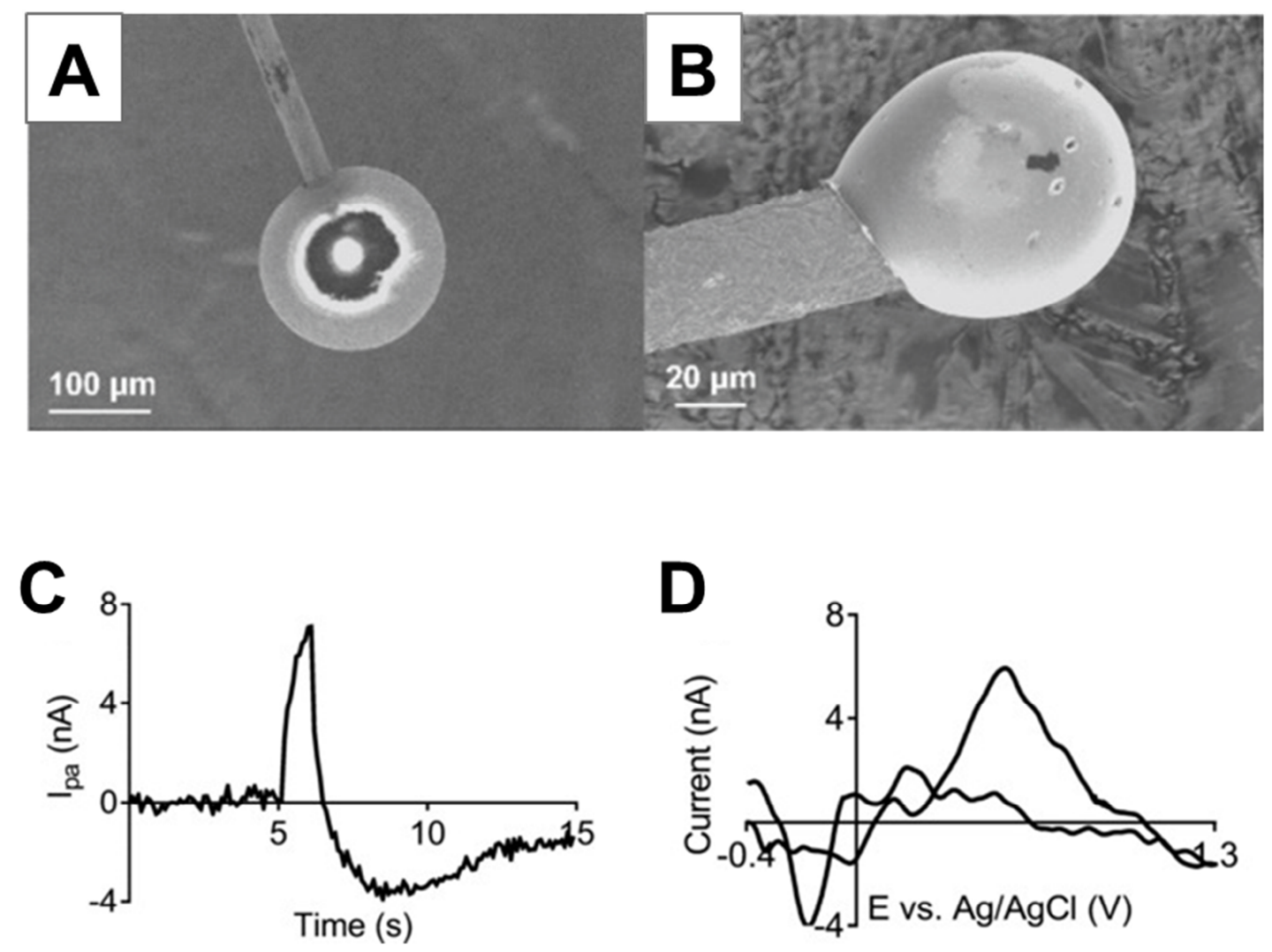
3D Printed Electrochemical Sensors: A new horizon for measurement of biomolecules

Bhavik Anil Patel ${ }^{1,2 *}$

${ }^{1}$ School of Pharmacy and Biomolecular Sciences and ${ }^{2}$ Centre for Stress and AgeRelated Diseases, University of Brighton, Brighton, BN2 4GJ, UK

${ }^{*}$ Corresponding authors

E-mail address: B.A.Patel@brighton.ac.uk

\section{Conflict of interest statement}

None 